\title{
The Application Development of Coiling Eccentricity Control Based on Wavelet Transform
}

\author{
Wenquan Sun*, Jian Shao, Anrui He and Quan Yang
}

Engineering Research Center for Advanced Rolling Technology, University of Science and Technology Beijing, Beijing 100083, China

\begin{abstract}
This paper proposes the eccentric problem in the coiling process of cold rolling mills. The wavelets multiresolution control method is designed: A wavelets multi-resolution controller is introduced in the control cycle of coiling tension control, whose purpose is to acquire the element caused by periodically change of the coiling eccentricity. Then the signal without effectiveness of the coiling eccentricity is feedback for coiling tension control. Therefore the precision of coiling tension control is improved. The Application Development of Coiling Eccentricity Control Based on Wavelet Transform.
\end{abstract}

Keywords: Coiling eccentricity, cold rolling, filter metod, wavelet transform.

\section{INTRODUCTION}

Coiler eccentricity or coil local convex might appear by the factors of equipment aging, processing pressing and special working condition like mill thread and start. For single stand cold rolling mill and conventional tandem cold rolling (single coil rolling), volume steels must be thread. When strip come into the gripper, local convex are formed on coil, and coil center is thick strip head, therefore, the coil center on the coiler is not round, which has certain eccentric and coil local convex.

In the rolling process, coiling eccentricity causes the cyclical fluctuation of strip tension at mill entrance or exit side. In the constant speed rolling process, the fluctuation cycle and range decrease as the coil diameter increases. The slight diameter fluctuation of coil has a great effect on tension, and will further affect the product quality.

\section{DESCRIPTIONS AND CONTROL METHODS OF COILING ECCENTRICITY}

\subsection{Descriptions of Winding Eccentricity}

In a broad sense, the coil diameter cyclical change caused by steel coil irregular on coiler is called coiling eccentricity. This problem will lead to strip thickness and tension periodically changes (Fig. 1).

Irregular shapes include the following respects,

(1) Eccentric e relative to the coiler drive center and the coil;

(2) Coil is oval instead of round;

(3) Coil diameter changes in a multiple cycle;
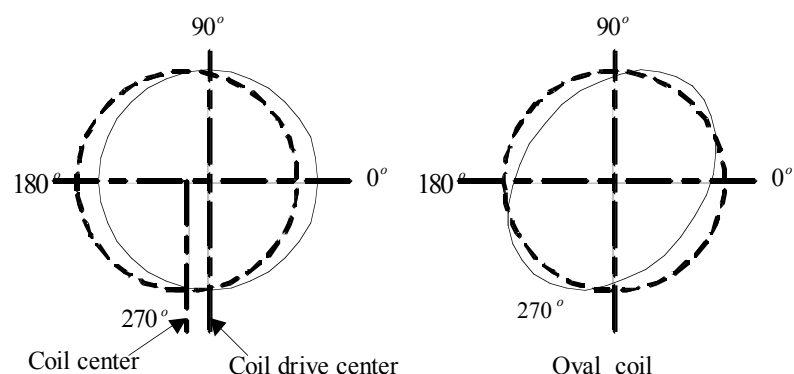

A: Single Cycle

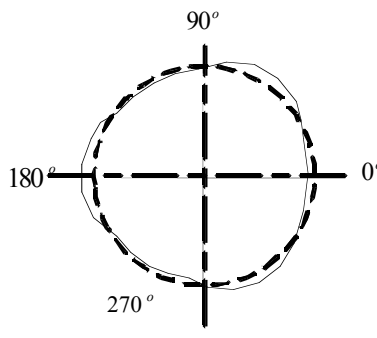

Multiple cycle eccentricity

C: Multi Cycle
Oval coil

B: Double Cycle

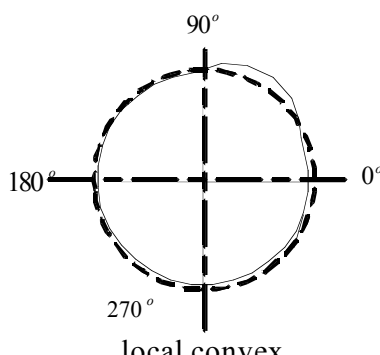

D: Local Convex

Fig. (1). Geometric relationship of coiling eccentricity.

(4) Coil diameter local convex;

Coiling eccentricity can be concluded to two basic types, namely the eccentricity caused by the difference between coiler drive center and coil center or the coil itself not round. Among the more common and with serious influence include thick strip start in pl-tandem cold rolling mill, or coil diameter sharp change when the thick strip is inserted into the gripper slot of coiler in conventional tandem cold rolling and single stand cold rolling. Of course, in actual cases coiling eccentricity may be the complex of those problems. So it seems that coiling eccentricity and roller eccentricity have similar forms $[1,2]$. 


\subsection{Analysis of Geometric Relationships of Coiling Ec- centricity}

(1) Eccentricity of core shaft and bear

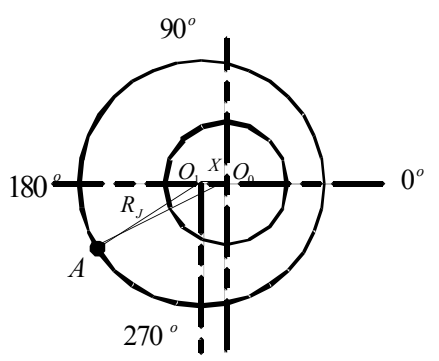

Fig. (2). Winding mandrel eccentricity heometric relationship.

$\mathrm{O}_{0}$ is the axis of the coiler core shaft (Fig. 2); $\mathrm{O}_{1}$ is the axis of coil; $\mathrm{X}$ is the distance between $\mathrm{O}_{0}$ and $\mathrm{O}_{1}$. Eccentricity trajectory is the rotation of point $\mathrm{A}$ around the point $\mathrm{O}_{0}$, which is expressed as $\mathrm{e}=\mathrm{O}_{0} \mathrm{~A}-\mathrm{R}_{\mathrm{J}}[3,4]$.

$\omega$ is angular velocity, then the angular $\varphi=\omega t$, in the triangle $\mathrm{AO}_{0} \mathrm{O}_{1}$, according to the cosine theorem:

$O_{0} A=\sqrt{\left(X^{2}+R_{J}^{2}-2 X R_{J} \cos \theta\right)}$

when the angular $\theta=180^{\circ}$, according to the sine theorem:

$\frac{\sin \omega t}{R_{J}}=\frac{\sin \left(180^{\circ}-\theta-\omega t\right)}{X}$

()()$=\frac{-\sin (\theta+\omega t)}{X}$

Then:

$-\sin (\theta+\omega t)=\frac{X}{R_{J}} \sin (\omega t)$

$\theta=\sin ^{-1}\left(-\frac{X}{R_{J}} \sin \omega t\right)-\omega t$

$-=-\sin ^{-1}\left(\frac{X}{R_{J}} \sin \omega t\right)-\omega t$

Therefore the parameter equation of eccentricity trajectory is:

$e=\sqrt{\left(X^{2}+R_{J}^{2}-2 X \cos \theta\right)}-R_{J}$

According to Equation 5, eccentricity waveform has been shown by (Fig. 3).

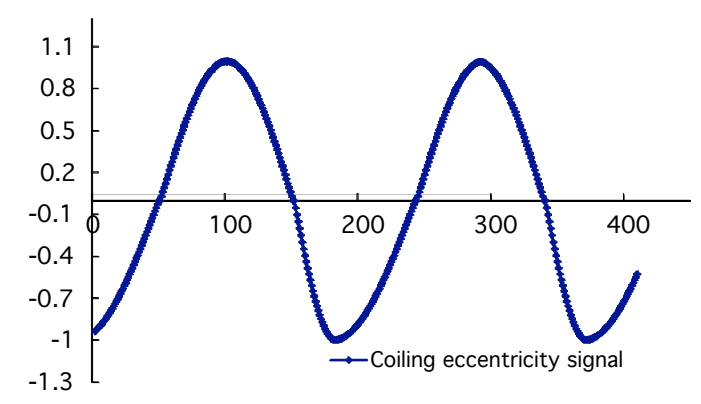

Fig. (3). Coiling tension eccentricity waveform graph.
(2) Coil oval form

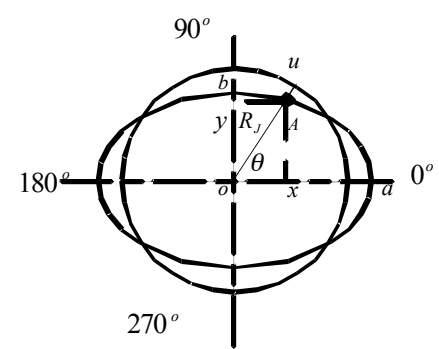

Fig. (4). Winding coil for oval diagram.

$\mathrm{O}$ is the axis of coiler; $\mathrm{R}$ is the ideal radius of coil, "a and b" are long and short axis of the ellipse (Fig. 4).

The eccentricity waveform is the trajectory that moveable point " $A$ " and the radial distance $e=R_{\mathrm{J}}-\mathrm{r}$ of ideal circumference, $r$ is the distance between point $\mathrm{A}$ and the axis. $\omega$ is the angular velocity, when $\mathrm{t}=0, \theta=0$, then:

$\left\{\begin{array}{l}x=r \cos \theta \\ y=r \sin \theta\end{array}\right.$

According to ellipse equation $\frac{x^{2}}{a^{2}}+\frac{y^{2}}{b^{2}}=1$ :

$\frac{r^{2} \cos ^{2} \theta}{a^{2}}+\frac{r^{2} \sin ^{2} \theta}{b^{2}}=1$

$\left(\frac{1}{b^{2}}-\frac{1}{a^{2}}\right) \sin ^{2} \theta=\left(\frac{1}{r^{2}}-\frac{1}{a^{2}}\right)$

$r=\frac{1}{\sqrt{\frac{a^{2}}{b^{2}} \sin ^{2} \theta+\cos ^{2} \theta}}$

The eccentricity curve is obtained:

$e=R_{J}-r=R_{J}-\frac{1}{\sqrt{\frac{a^{2}}{b^{2}} \sin ^{2} \theta+\cos ^{2} \theta}}$

The eccentricity curve is similar to the sine function (Fig. 3).

\section{MATHEMATICAL MODEL ANALYSIS OF TEN- SION FLUCTUATING CAUSED BY COILING EC- CENTRIC}

Theoretical analysis and real production historical data analysis show: coiling eccentric is reflected on tandem cold mill export tension and strip thickness, and is similar to highfrequency periodic wave. Eccentric signal frequency depends on rolling speed and coil diameter, and amplitude depends on coiling eccentricity and coil diameter. The existence of eccentric directly leads to cyclical changes of cold rolling unit export tension, and causes fluctuations in the strip thickness. Tensile variation can be expressed as the function of total coiling eccentricity. Rotation of the coil eccentric causes exports tension periodical change approximately. 


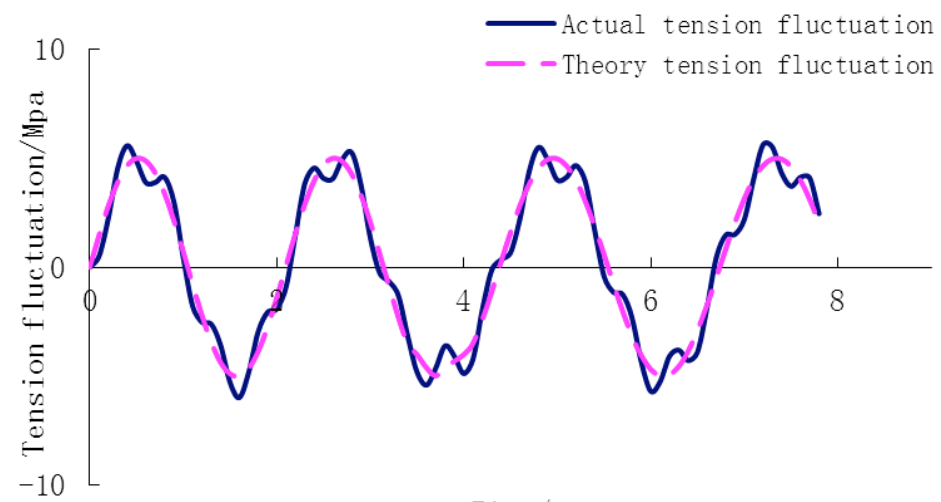

Time/s

Fig. (5). Tension fluctuation of winding mandrel eccentricity.

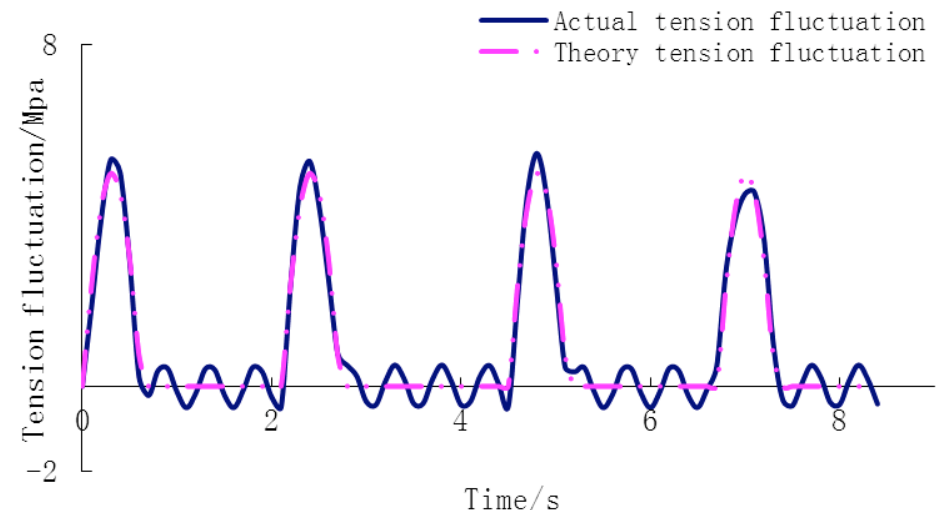

Fig. (6). Tension fluctuation of winding local convex.

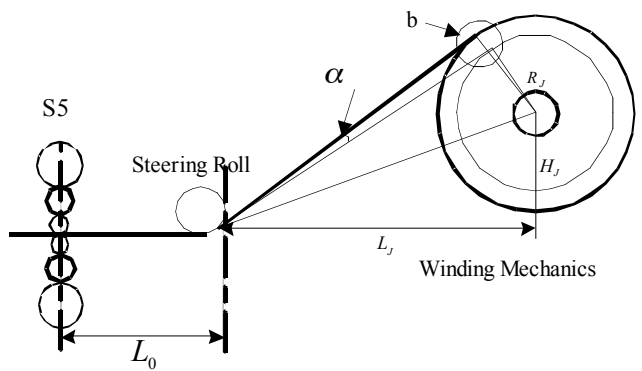

a

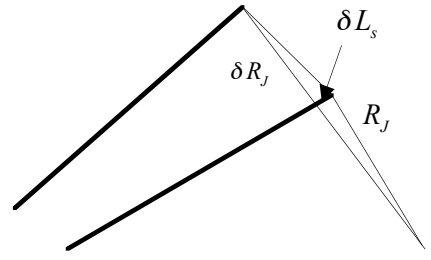

b

Fig. (7). Coiler diameter fluctuations on the coiling tension relationship diagram.

When rolling at a constant speed, coil diameter becomes larger progressively, and the angular velocity of the winder is gradually reduced, in this way, the strip export tension fluctuation cycle increases gradually, say $\mathrm{T}_{2}>\mathrm{T}_{1}$ (Fig. 5), obviously fluctuations of unit export tension periodic changes, similar to non-sinusoidal signals.

Local salience caused by coil tension fluctuations (Fig. 6) shows. Tension fluctuation signal has the characteristics of pulse changes, fluctuations are severe, cycle of tension fluctuations increases gradually.

The impact of coiling eccentric is mainly reflected in the impact of the coiling tension, tension fluctuations influences the rolling force, thus impacts on the thickness of the rolling.

According to the geometric relationships between coiling coils on the machine and export strip (Fig. 7), calculate changes in export strip extension length caused by coil diameter changes as Eqs. (11) shows:

$$
\begin{aligned}
\delta L_{S} & =\sqrt{L_{J}^{2}+H_{J}^{2}-\left(R_{J}+\delta R_{J}\right)^{2}} \\
& -\sqrt{L_{J}^{2}+H_{J}^{2}-R_{J}^{2}}
\end{aligned}
$$

According to commonly used strip tension and elongation relationship Eqs. (12), further calculate fluctuations in the coiler volume diameter to strip tension impact relationships Eqs. (14).

$$
\begin{aligned}
& \frac{d \tau_{5}}{d t}=\Delta \varepsilon \cdot E \\
& \Delta \varepsilon=\delta L_{S} /\left(\sqrt{L_{J}^{2}+H_{J}^{2}-R_{J}^{2}}+L_{0}\right)
\end{aligned}
$$


$\delta \tau_{f 5}^{\prime}=E \cdot \Delta \varepsilon$

$\delta L_{S}$ - strip extension length change caused by volume diameter fluctuations, $\mathrm{m} ; L_{\mathrm{J}}$-horizontal distance from the steering roller to the volume the heart of the coiler, $\mathrm{m}$;

$L_{0}$ - the distance of the mill exports to the steering roller, $\mathrm{m}$; HJ-the vertical distance of the coiler volume of the heart relative to the steering roller, $\mathrm{m} ; \Delta \varepsilon$ - change of the mill exports to the coiling machine strip elongation.

Besides, as coiling machine uses torque loop control, when torque keeps constant and coil diameter changes, the coiling tension will change the corresponding, change value is as Eqs. (15) shows, the total coiling tension variation is as Eqs. (16) shows.

$$
\begin{aligned}
\delta \tau_{f 5}^{\prime \prime} & =\frac{T_{J}}{R_{J}+\delta R_{J}}-\frac{T_{J}}{R_{J}} \\
& =-\frac{\delta R_{J} T_{J}}{R_{J}\left(R_{J}+\delta R_{J}\right)} \\
\delta \tau_{f 5} & =\delta \tau_{f 5}^{\prime}+\delta \tau_{f 5}^{\prime \prime}
\end{aligned}
$$

From Eqs. (12) and Eqs. (13) we can see that, the larger the coil diameter is, the greater are the fluctuations of the tension coil diameter. In the rolling process, coiler tension loop control system can slow down the fluctuations of the coil diameter of tension and thickness, however, coiler tension loop is a feedback control, which has certain hysteresis, and the coiling machines and steel coil are of great inertia, coiler tension closed loop has a limited ability of eliminating tension and thickness fluctuations caused by coiling center being not round, especially when mill running at a high speed, coiler tension closed loop basically does not work on coiling eccentric or not round.

On the other hand, when coiler uses direct tension control, the tonometer detected coiling tension signal is generally required to be filtered, and the tension signal after filtering is considered to be the actual detection value of the direct tension closed-loop control. Through this process, direct tension control has lost the ability to control the take-up eccentric, the ability gradually reduces as the rolling speed increases. For indirect tension control of the coiling control system, it has no eccentric coiling corrective ability.

\section{APPLICATION OF COILING ECCENTRICITY CONTROL BASED ON WAVELET TRANSFORM}

The essence of detecting eccentric signals lies in eliminating all kinds of noise contained in detected signals, separating the eccentric signals and taking the appropriate control strategy to reduce the effect of eccentricity on system [5].

Eccentricity signal in the coiling process behaves as a high-frequency periodic wave hidden in the winding tension and the strip thickness. According to the signal processing theory, the conventional method to identify the eccentricity signal is the Fast Fourier Transform (FFT). However, the FFT-based identification algorithm has a localized conflict between time domain and frequency domain, which limits its application to spectrum analyzing the signals clearly distin- guished from the noise, rather than to separate the highfrequency eccentricity signal from the random noise in the coiling process. Thus, the signal denoising results are poor.

The wavelet transform, developed from Fourier transform, has the character of time-frequency localization. As a better signal processing method than the traditional Fourier transform, the wavelet transform can decompose a signal to signals in different frequency band, reconstruct the signal from the decomposed results selectively, and so realize the signal-noise separation. Therefore, the winding tension signal and the thickness signal could be separated effectively from the random noise, and the accurate signal could be obtained for the eccentric control in coiling process.

In this paper, the winding tension signal and the thickness difference signal are analyzed by the wavelet transform algorithm. The main idea is to utilize the characteristic of ... to separate the signal from random noise, thus get a better eccentric signal and carry out a more accurate control in coiling process.

\subsection{Denoising Algorithm with Wavelet}

The use in signal denoising is an important applications of wavelet transform $[2,6,7]$. A one-dimensional signal model with noise could be expressed as:

$y\left(t_{i}\right)=f\left(t_{i}\right)+\sigma_{Z i} i=1,2, \ldots, \mathrm{n}$

$f\left(t_{\mathrm{i}}\right)$ represents the real signal, $Z_{\mathrm{i}}$ is noise, $\sigma$ is noise intensity and $t_{\mathrm{i}}$ is the sampling time. Decompose the noised signal with wavelet (for example, 3 layers decomposition):

$$
\begin{aligned}
S & =c A_{1}+c D_{1}=c A_{2}+c D_{2}+c D_{1} \mathrm{i}=1 \\
& =c A_{3}+c D_{3}+c D_{2}+c D_{1}
\end{aligned}
$$

$c A_{\mathrm{i}}$ is the approximate part, and $c D_{\mathrm{i}}$ is the detail part.

Generally, the noise is contained in $c D_{1}, c D_{2}, c D_{3}$. By using the threshold to process the wavelet coefficients, and reconstructing the signal, then the purpose of denoising could be achieved $[8,9]$.

If the signal's maximum amplitude of the local module from wavelet is rapidly increasing while the scale is decreasing, it means that the singularity is mainly controlled by noise. Therefore, this noise should be removed in denoising process. The detailed steps are as follows [10]:

(1) Use Mallat fast algorithm to calculate the noised signal's binary wavelet transform in each scale $j(j=1,2 \ldots J$, generally $\mathrm{J} \leq 4$ ):

$$
\begin{aligned}
& S_{2^{j}} f(x)=\sum_{l \in Z} h_{l} S_{2^{j-1}} f\left(x-2^{j-1} l\right) \\
& W_{2^{j}} f(x)=\sum_{l \in Z} g_{l} S_{2^{j-1}} f\left(x-2^{j-1} l\right)
\end{aligned}
$$

(2) Find the critical value $T_{0}=C \frac{|V|}{J}$

$\mathrm{M}$ is maximum amplitude in the maximum scale $2^{J}, \mathrm{C}$ is a constant. 

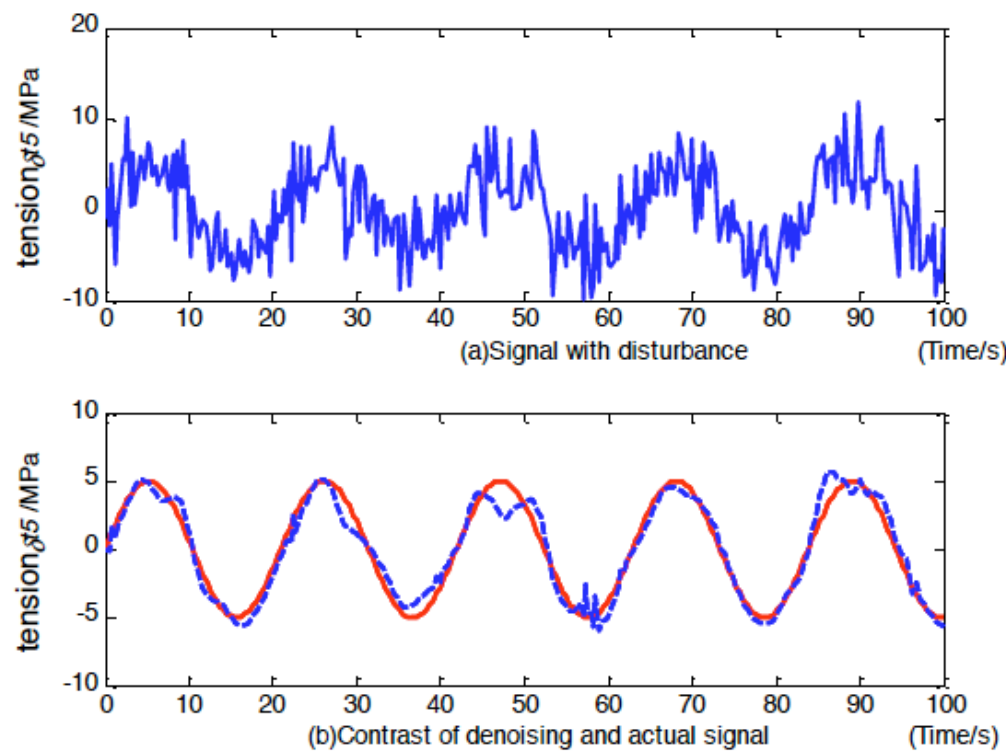

Fig. (8). Wavelet denoising effect of tension interference signal.

(3) In the last scale, set the wavelet transform to zero if $\left|W_{2^{j}} f(x)<T_{0}\right|$. Otherwise, approximately seek the Lipschitz exponent of the corresponding point $\mathrm{x}$ :

$a=\log _{2}\left|\frac{W_{2^{j+1}} f(x)}{W_{2^{j}} f(x)}\right|$

Generally, $\mathrm{j}=3$ or 4 . If $\alpha<0$ (or $\alpha<-0.5$ ) at a point $\mathrm{x}$, then set $\alpha=0$.

(4) Remove all the wavelet transform at scale $1,2, \ldots, \mathrm{J}-1$.

(5) Use the wavelet transform at the last scale, i.e. $\mathrm{j}=\mathrm{J}$, and $W_{2^{j}} f(x)=W_{2^{j+1}} f(x) 2^{-\alpha}$ to reconstruct the wavelet transform at scale $\mathrm{j}=\mathrm{J}-1 \ldots, 1,2$

(6) Restore the cleaned signal from the reconstructed wavelet transform by invert wavelet transform.

$$
\begin{aligned}
S_{2^{j-1}}^{d} f(x)= & 2 \sum_{l=-\infty}^{l=\infty} h(x-2 l) S_{2^{j}}^{d} f(x) \\
& +2 \sum_{l=-\infty}^{l=\infty} g(x-2 l) W_{2^{j}} f(x)
\end{aligned}
$$

In general, the noises are contained in the high-frequency details. Thus, the threshold methods could be adopted to process the decomposed wavelet coefficients, and then the signal could be reconstructed to achieve the purpose of noise reduction

\subsection{Simulation analysis of Coiling Eccentricity Control Based on Wavelet Transform}

Take the coil's radius as $0.7 \mathrm{~m}$, mix in the a sine function with a amplitude $\delta R_{J}=0.01 R_{J}$ as the coil's diameter fluctuations, and mix in random disturbance signal, which follows a normal distribution whose variance is $3 \mathrm{Mpa}$ and standard deviation is $\sigma=1$. Use the One-dimensional wavelet denoising (Fig. 8).
From the simulation results (Fig. 8), we can see that the amplitude of disturbance signal before processing is significant, which reaches the same amplitude of the real eccentric signal. The disturbance signal is very similar with the tension fluctuation signal in reality. Consequently, the real eccentric signal could be reconstructed by the wavelet method, which provides a more accurate real-time signal to carry out the eccentricity control in coiling process.

\section{CONCLUSION}

(1) The characteristics and geometric relationships of coiling eccentricity have been fully analyzed in this paper; and the characteristics of signal due to the fluctuation of coiling tension and thickness have been analyzed.

(2) The model reflecting the influence eccentricity exerts on tension has been built, then the dynamic effects eccentricity exerts on the unit have been analyzed. Simulation results show that the fluctuation of thickness and profile due to the eccentricity has to be taken into consideration in high precision cold rolling production.

(3) Wavelet transform method has been used to eliminate the noise from the signal. In this way, the high frequency interference signal has been filtered, ensuring an accurate signal for the control of eccentricity.

\section{CONFLICT OF INTEREST}

The authors confirm that this article content has no conflict of interest.

\section{ACKNOWLEDGEMENTS}

This work is supported by Doctoral Program Foundation of Institutions of Higher Education of China (No. 20130006120024).

\section{REFERENCES}

[1] W.R. Wang, Roll Eccentricity Research on Cold rolling Using Advanced Control Theory and Hardware Application, University of Science and Technology Beijing, China, 2001. 
[2] N.F. Protmann, D Lindhoff, G Sörgel, O Gramckow, "Application of neural network in rolling mill automation," Iron and Steel Engineer, vol. 72, no. 2, pp. 33-36, 1995.

[3] T. Iwashita, Y. Suzuki, T. Sakai, "New roll eccentricity compensation system", Furukawa Review, vol. 26, no. 6, pp. 117$119,1993$.

[4] Z.Y. Wang, J. Wang, and G.D. Wang, "A new method of filter of rolling force and compensation of rolling gap", Research on Iron \& Steel, vol. 1, pp. 142-145, 2002.

[5] L.Z. Ceng, H.X. Wang, and Y. Luo, Theory and Application of Wavelet, Science Press, Beijing, 2004. (In Chinese).

[6] Z.X. Cheng, S.Z. Yang, and X.X. Feng, Wavelet Analysis Theory, Algorithms, and Application Progress, National Defence Industry Press, Beijing, 2007.
[7] Y. Zhang, X.H. Liu, and G.D. Wang, "Roll eccentricity analysis and simulation based on wavelet transform", Journal of Northeastern University (Natural Science), vol. 25, no. 7, pp. 671-673, 2004 (In Chinese).

[8] Y.H. Zhang, X.H. Liu, and G.D. Wang, "Wavelet transform and its application in roll eccentricity," Research on Iron \& Steel, vol. 18, no. 1, pp. 11-13, 2005

[9] Y.B. Deng, Z.H. Tan, Y. Huang, "A study about the influencing factors for wavelet de-noising ", Journal of East China Jiaotong University, vol. 22, pp. 161-164, April 2005.

[10] Z. X. Ge, W. Sha, Wavelet Analysis Theory and Implementation. Bei Jing: Electronic Industry Press, Beijing, 2007.10.

Received: September 22, 2014

Revised: November 30, 2014

Accepted: December 02, 2014

(C) Sun et al.; Licensee Bentham Open.

This is an open access article licensed under the terms of the Creative Commons Attribution Non-Commercial License (http://creativecommons.org/licenses/by-nc/3.0/) which permits unrestricted, non-commercial use, distribution and reproduction in any medium, provided the work is properly cited. 